\title{
Treating Late-Time Instability of Partially Tetrahedral-Gridded Finite Difference Time Domain Method
}

Chieh-Tsao Hwang* and Ruey-Beei $W_{u}$

Department of Electrical Engineering, Rm. 340, National Tajwan University Taipei, Taiwan, 10617, Republic of China

\section{Summary}

A partially tetrahedral-gridded FDTD method has recently been proposed to solve the time-domain electromagnetic scattering of two or three-dimensional arbitrarily shaped dielectric objects [1],[2]. It successfully employs the conventional FDTD method for most of the regular region but introduces the tetrahedral edgebased finite element scheme to model the region near the arbitrarily curved surfaces. Numerical results of early time simulation validate that the method has the advantages of accuracy, flexibility, stability, and computational efficiency. For the analyses of electromagnetic problems of high $Q$ systems, transient response over a very long duration is necessary. It is found that the simulation may suffer from late-time instability and spurious dc modes. This paper is focused on treating the two drawbacks of the hybrid method.

A. Temporal Filtering Technique for Late Time Instability

The strategy employed here is to derive a low pass filter to suppress the computational error which is of higher frequencies, while keeping the low frequency components nearly unchanged. The filter corresponds to an IIR system defined by [3]

$$
F(n)=p \cdot f(n+1)+(1-2 p) \cdot f(n)+p \cdot F(n-1)
$$

where $F$ and $f$ denote the filtered and original fields, respectively. The positive constant $p$ is chosen to determine the attenuation of the filter at higher frequencies.

To verify the performance of this filter, the relation (1) with $p=0.05$ is applied to modify the electric fields at time step $n$ in entire FEM region after obtaining the fields at time step $n+1$ from the FEM solver. Fig.l shows the simulation result modified by the temporal filtering technique for the three dimensional dielectric sphere scattering problem considered in [2]. In the early time response shown in Fig.1(a), the desired signal is hardly affected by the enforcement of this filter. In the late time response shown in Fig.1(b), it is verified that the instability due to the the late time response shown in Fig.1(b), it is verified that

B. Frequency Shifting Technique for Spurious Curl-Free Mode

A magnified view of the filtered late time response in Fig. I(b) reveals that there are spurious components which grow linearly with time. The occurrence is replatted as the solid curve in Fig. 2 where the vertical axis is in a different scale from Fig1.(b). This spurious mode can be attributed to the insufficiency of the equation 


$$
\nabla \times \nabla \times \vec{E}+\mu \epsilon \frac{\partial^{2} \vec{E}}{\partial t^{2}}=0
$$

to model the Maxwell's equations at the dc limit. It is no difficult to show that (2) has a nontrivial solution of the form

$$
\vec{E}_{\imath}=-\dot{\nabla} \phi \cdot t
$$

for arbitrary scalar function $\phi(\vec{r})$. This means that any curl free distribution once generated, no matter how small, will grow up to be significant in the very late time.

One way to destroy the linear growth of the spurious modes is to add a dissipative time $\delta \vec{E}$ to the left hand side of (2). The corresponding nontrivial solution becomes

$$
\bar{E}_{\mathrm{s}}=-\nabla \phi \cdot \cos \left(\omega_{\mathrm{\delta}} t\right)
$$

with an angular frequency of $\omega_{\delta} \equiv \sqrt{\frac{\delta}{\mu t}}$. This field pattern remains small as time marches.

The best choice of $\delta$ will be the smallest one which is able to suppress the linear growth of the spurious modes. Numerical experiments have bcen evoked to find this $\delta$. Fig. 2 shows the simulation results with $\delta=4 \times 10^{-6}$ and $4 \times 10^{-7} \frac{\mu t}{\Delta^{2}}$ as a parameter. After an extensive trial, it is found that $\delta=4 \times 10^{-6} \frac{\mu c}{\Delta_{l}^{?}}$ is enough to destroy the linear growth of spurious modes.

\section{Numerical Results}

The hybrid FDTD together with the aforementioned stabilizing approach is applied to deal with the dielectric resonators (DR). The example considers an isolated DR. The DR is made of the dielectric with $\epsilon_{r}=38$ and has the geometry parameters of radius $a=5.25 \mathrm{~mm}$ and height $h=4.6 \mathrm{~mm}$.

The recorded signals can be represented by the summation of several damped sinusojdal functions, each of which denotes a mode. The number of significant modes and the modal parameters, i.e., the resonant frequency and the $Q$ factors, can be extracted from the recorded responscs by the matrix pencil technique [4]. The results are listed in Table.1 and compared with those by other methods, e.g., the method of line, the method of moments, the null-field method, two dimensional FDTD in angularly symmetric structures $[5$, and the references therein], as well as the measurement results [6]. The good agreement validates the correctness of the present method.

Finally, the present method is applied to calculate the scattering parameters on the microstrip line due to the presence of the cylindrical dielectric resonator. In the simulation, the same DR is mounted with a microstrip line of substrate dielectric constant $\epsilon_{r}=2$. The structure is enclosed by the metallic walls except the input and output sides of the microstrip lines, where the PML are placed to absorb the scattered fields. An excitation source with modulated Gaussian waveform is incident to one end of the microstrip line.

Fig. 3 shows the resultant scattering parameters versus frequency obtained from the responses by inverse FFT. Please notice the drastic variations near the resonant frequencies of the DR modes. Among the modes recognized in Table.1, the $T E_{01 s}$ mode exhibits strongest coupling with the microstrip line. The hybrid modes $H E M_{11 s}$ and $H E M_{12 s}$ also appear noticeable effects. However, the $T M_{01 s}$ mode of DR seems to be orthogonal with the guided microstrip line mode and consequently, no reflected field is observed at its resonant frequency. 


\section{References}

[1] R. B. Wu and T. Itoh, "Kybridizing FD-TD analysis with unconditionally stable FEM for objects of curved boundary," IEEE MTT-S 1995 Int. Microwave Symp. Dig., pp.833-836, May 1995.

[2] R. B. Wu and T. Itoh, "Hybrid finite-difference time-domain modeling of curved surfaces using tetrahedral edge elements," IEEE Trans. Antennas Propagat., vol. 45, pp.1302-1309, Aug. 1097

[3] D. J. Riley and C. D. Turner, "VOLMAX: A solid-model-based, transient volumetric Maxwell solver using hybrid grids," IEEE Antennas and Propagation Magazine, vol. 39, pp.20-33, Feb. 1997.

[4] Y. B. Hua and T. K. Sarkar, "Generalized pencil-of-function method for extracting poles of an EM system from its transient response," IEEE Tans. Anternas Propagal, vol 37. pp.229-234, Feb. 1989.

[5] D. Kremer and R. Pregla, "The method of lines for the hybrid analysis of multilayered dielectric resonators," IEEE MTT-S 1995 Int. Microwave Symp. Dig., pp.491-494, May 1995

[6] R. K. Mongia, C. L. Larose, S. R. Mishra, and P. Bhartia, "Accurate measurement of Q factors of isolated dielectric resonators," IEEE Trans. Mirrowave Theory Tech., vol. 42. pp.1463-1467, Aug. 19:4

Table.1 Comparison of the calculated resonant frequencies and $Q$ factors of various resonant modes in an isolated DR by several different methods.

\begin{tabular}{|c|c|c|c|c|}
\hline \multirow[t]{2}{*}{ Method } & \multicolumn{4}{|c|}{$k_{0} a$ of resonant modes } \\
\hline & $T E_{018}$ & ${I I E M_{11 \delta}}$ & $H E M_{12 \delta}$ & $T M_{01 \delta}$ \\
\hline Mol & 0.537 & 0.696 & 0.732 & 0.827 \\
\hline MoM & 0.531 & 0.696 & $0 . \overline{730}$ & 0.827 \\
\hline Null-field & 0.534 & 0.698 & 0.731 & 0.829 \\
\hline 2D FDTD & 0.535 & 0.698 & 0.731 & 0.827 \\
\hline Measurement & 0.533 & $0 . \overline{696}$ & 0.726 & 0.824 \\
\hline Present method & $0 . \overline{534}$ & 0.698 & 0.728 & 0.823 \\
\hline
\end{tabular}

\begin{tabular}{|c|c|c|c|c|}
\hline \multirow{2}{*}{ Method } & \multicolumn{4}{|c|}{ Q-factor of resonant modes } \\
\cline { 2 - 5 } & $T E_{01 \delta}$ & $H E M_{11 \delta}$ & $H E M_{12 \delta}$ & $T M_{01 \delta}$ \\
\hline MoL & 43.73 & 31.46 & 48.55 & 72.96 \\
\hline MoM & 45.8 & 30.7 & 52.1 & 76.8 \\
\hline Null-field & 40.80 & 30.85 & 50.30 & 76.90 \\
\hline 2D-FDTD & 47 & 31 & 46 & 71 \\
\hline Measurement & 46.4 & 30.3 & 43.3 & 58.1 \\
\hline Present method & 36.81 & 28.81 & 49.42 & 77.72 \\
\hline
\end{tabular}




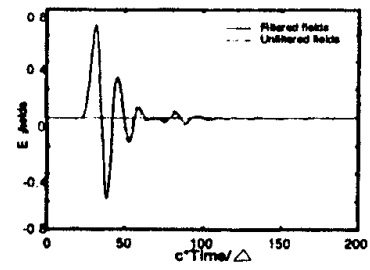

(a)

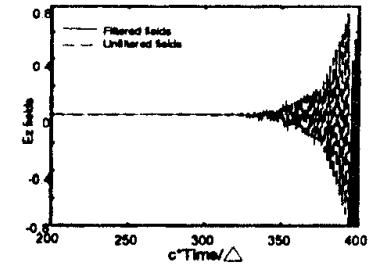

(b)

Fig.1 (a) Comparisons between filtered and original fields in early time responses. (b) Comparisons between filtered and original fields in late time responses.

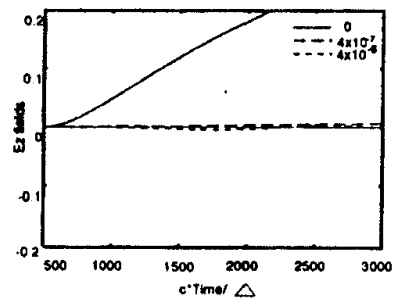

Fig. 2 Effects of the frequency shifting on the filtered response shown in Fig.1. The shift value $\delta$ is chosen as 0 (no shift), $4 \times 10^{-7} \frac{\mu e}{\Delta_{1}^{2}}$, and $4 \times 10^{-6} \frac{\mu e}{\Delta_{i}^{2}}$.
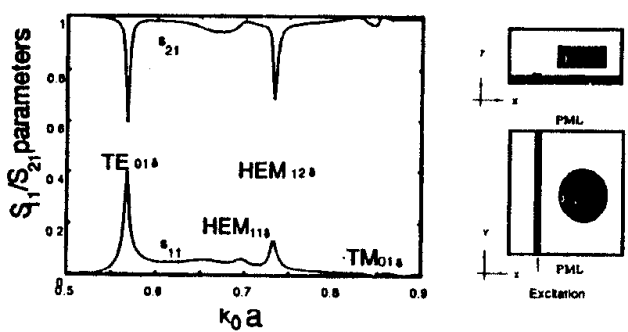

Fig.3 Reflected and transmitted coefficients versus normalized wave number $\left(k_{0} a\right)$. 